\title{
A Low Complexity Algorithm for Detecting Rotational Symmetry Based on the Hough Transform Technique
}

\author{
Soo-Chang Pei and Ji-Hwei Horng \\ Department of Electrical Engineering \\ National Taiwan University \\ Taipei, Taiwan, R.O.C. \\ Email: pei@cc.ee.ntu.edu.tw
}

\begin{abstract}
The Hough transform technique has been applied to detect rotational symmetry by Raymond K.K. Yip [1]. He proposed an $\mathcal{O}\left(N^{3}\right)$ algorithm, where $N$ is the number of edge points in the input image, to extract global or local features of rotational symmetry in the presence of noise and occlusion. In this paper, we follow the Yip's concept and design a simple and efficient algorithm, which can detect the rotational symmetry in $\mathcal{O}\left(N^{2}\right)$ time. Some simulation results of the new proposed algorithm are also given.
\end{abstract}

\section{Introduction}

Ratational symmetry is an important property of a shape. If certain parts of a rotationally symmetric shape(abbreviated as RSS henceforth) are missing, the shape can be recovered by using the property of symmetry without the need of models. Another benefit of rotational symmetry is that any processing of the RSS can be done by computing one of the repeating portions. Besides, the storage of a RSS requires to store only one repeating portion.

Many methods have been proposed to detect the number of folds and the orientation of a RSS. Tsai proposed the generalized principal axes of a RSS[2]. The principal axis of a shape is defined as the line with respect to which the moment of inertia is minimized[3]. For a RSS, no principal axis can be determined. Its moment of inertia is a constant with respect to any line going through its centroid. The generalized principal axes of a $n$-fold RSS are defined, by Tsai, as the lines with respect to which the $n$ th-order central moment of inertia is minimized. Pei proposed an algorithm to detect the number of folds and to normalize the RSS[4]. The Fourier descriptor of a $n$-fold RSS has nonzero values only at multiples of $n$. The first nonzero Fourier coefficient is detected and the shape is rotated properly so that the phase of this coefficient becomes zero. Lin proposed a simple mathematical property[5],[6]. Which is used to detect the number of folds and to define the universal principal axes of a RSS.

The application of Hough technique to the detection of RSS is first proposed by Yip[1]. His algorithm can handle shapes formed by separate points containing missing data due to noise or occlusion. This property of the Hough technique is attractive, since the other methods usually can only handle shapes with simple closed contours. However, the Hough technique has the disadvantage of high computational complexity. Yip reduced the dimensionality of the parameter space by breaking it down into three passes. In this paper, we propose an algorithm which is more efficient than the Yip's and enables the Hough technique to detect RSS within reasonable time.

\section{Proposed algorithm}

An $n$-fold RSS has the property that if it is rotated around its centroid by any multiple angle of $2 \pi / n$, the rotated version is identical to the original shape. That is, there are lots of similar isosceles triangles, each of them is formed by a pair of edge points with the center of rotation and has an angle $2 \pi / n$ at the center of 
rotation. For such triangles, the median that bisects the side joining edge points is a perpendicular bisector and the ratio of the side to the median is $2 \tan (\pi / n)$. Figure 1(a) shows an 8-fold RSS. Some of the similar isosceles triangles are shown in Figure 1(b).

The Hough technique[7],[8] can be applied to detect RSS based on the above properties. If the number of folds is given, two possible centers of rotation are hypothesized and voted in the parameter space for each pair of edge points. Then, the peak position in the parameter space is detected as the center of rotation. Given edge points of the input image $S=\left\{\left(x_{1}, y_{1}\right),\left(x_{2}, y_{2}\right), \ldots,\left(x_{N}, y_{N}\right)\right\}$, the proposed algorithm is summarized as follows:

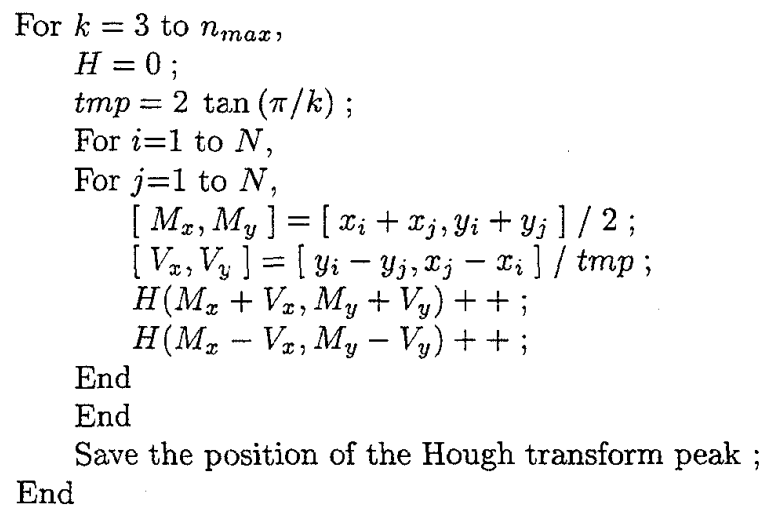

where $H$ is the accumulator array of the Hough transform and $n_{\max }$ is the maximum number of folds of the RSS expected to be detected.

Since only local feature of RSS is used, the algorithm can be applied to detect partial shapes. If there are multiple RSS, there will be multiple local peaks in the parameter space. The algorithm can also resolve concentric RSSs with different numbers of folds, because RSSs with different numbers of folds are detected at different iterations $(k$-th iteration for detecting $k$-fold RSS in the algorithm). That is, the Hough technique can be applied to detect mutually occluded shapes in a noisy scene.

Notice that, an $n$-fold RSS is also a $k$-fold RSS provided that $n / k \in$ integer. Therefore, if the number of folds $n$ is not a prime number, multiple peaks with a common center of rotation will present at different iterations. The number of folds which divided by the others is defined as the number of folds of such an RSS.

The presence of Hough transform peak only ensures that there are lots of point pairs fit the local feature. However, it says nothing about the global structure of the detected RSS. The global structure of the detected RSS can be verified by the procedure proposed in the third pass of the Yip's algorithm. Given the edge image and the set of edge points $S$, the verification of the detected center of rotation $O$ and number of folds $n$ is summarized as follows:

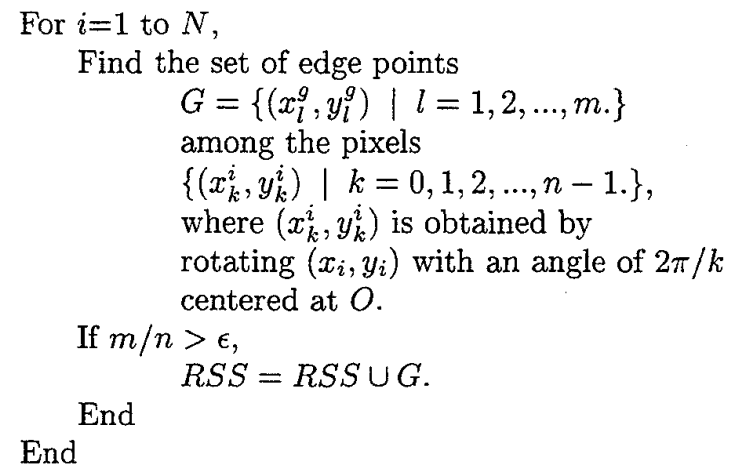

where $\epsilon$ is a threshold which defines the least percentage of total folds, of the detected RSS, expected to be seen in the resultant image. The point set $R S S$ extracted from the input image is the detected RSS corresponding to the center of rotation $O$ and the number of folds $n$.

\section{Experimental results}

Our algorithm is programmed in MATLAB language and run on the Sun SPARC 10 workstation. Figure 2(a) is the $60 \times 60$ digital image of a 5 -fold RSS. The Hough transform data of the iteration $k=5$ is plotted in Figure 2(b), where a peak is presented at the center of the star. The vote counts at the same position corresponding to different iterations are plotted in Figure 2(c). Noticing that, the vote counts plotted in Figure 2(c) are normalized by the factor $\tan (\pi / k)$.

In our algorithm, two accumulators are incremented by each pair of edge points. If the distance between the pair of edge points deviates $\tan (\pi / k)$ pixels, the same accumulators will be incremented due to the quantization effect of the discrete accumulator array. That is, the number of edge point pairs which increment the same accumulator is approximately proportional to the factor $\tan (\pi / k)$. The vote counts plotted in Figure 2(c) are normalized by this factor.

Figure $3(\mathrm{a})$ is the $60 \times 60$ digital image of a 6 -fold RSS. The Hough transform data of the iteration $k=6$ is plotted in Figure 3(b). Figure 3(c) shows the vote counts of different iterations at the peak position of Figure $3(\mathrm{~b})$. There is a peak at the iteration $k=3$, 
since the number of folds 6 is divided by 3 .

To demonstrate the applicability of the Hough technique to the occluded shapes in noisy scene, our algorithm is applied to the $70 \times 70$ digital image of occluded RSSs shown in Figure $4(a)$. Figure $4(b)$ and 4 (c) show the Hough transform data of the iterations $k=3$ and 5 respectively. The vote counts of different iterations at the peak positions of Figure $4(\mathrm{~b})$ and 4(c) are plotted in Figure 4(d) and 4(e) respectively.

\section{Complexity}

The Yip's algorithm has computing time $\mathcal{O}\left(N^{3}\right)$, since the most time consuming pass (the third pass) of the algorithm has computing time $\mathcal{O}\left(N^{3}\right)$. Our algorithm has computing time $\mathcal{O}\left(N^{2}\right)$. Although the basic operations of the two are not exactly the same, our algorithm is much faster than the Yip's. Of course, the same amount of memory are used in the both algorithm.

For example, in applying to the image shown in Figure 2(a), the first pass of the Yip's algorithm requires 5069 CPU time in seconds: however, our algorithm requires only $226 \mathrm{CPU}$ time in seconds. If they are applied to a larger image with more edge points, the CPU time consumed by the Yip's algorithm will grow faster than that consumed by our algorithm.

\section{Conclusion}

In this paper, we propose an $\mathcal{O}\left(N^{2}\right)$ algorithm to detect rotationally symmetric shapes. The Hough technique is applied to detect the center of rotation and the number of folds of the RSS by using local features. Then, the RSS is extracted from the input image based on the detected parameters. Since only local features are used, the algorithm can handle occluded shapes under noisy environment. Experimental results demonstrate the usefulness of our algorithm.

\section{References}

[1] Raymond K.K. Yip, Wilson C.Y. Lam, Peter K.S. Tam, and Dennis N.K. Leung, A Hough transform technique for the detection of rotational symmetry, Pattern Recognition Letters 15. pp. 919-928, 1994.

[2] Wen-Hsiang Tsai and Sheng-Lin Chou, Detection of generalized principal axes in rotationally sym- metric shapes, Pattern Recognition, Vol. 24, No. 2, pp. 95-104, 1991.

[3] A. Rosenfield and A. C. Kak, Digital Picture Processing, Vol. II, Academic Press, New York, 1982.

[4] Soo-Chang Pei and Chao-ivan Lin, Normalization of rotationally symmetric shapes for pattern recognition, Pattern Recognition, Vol. 25, No. 9, pp. 913-920, 1992.

[5] Ja-Chen Lin, Universal principal axes: an easy-toconstruct tool useful in defining shape orientations for almost every kind of shape, Pattern Recognition, Vol. 26, No. 4, pp. 485-493, 1993.

[6] Ja-Chen Lin, Wen-Hsiang Tsai, and Jun-Ann Chen, Detecting number of folds by a simple mathematical property, Pattern Recognition Letters 15, pp. 1081-1088, 1994.

[7] Jack Sklansky, On the Hough technique for curve detection, IEEE Trans. Comput., Vol. c-27, No. 10, pp. 923-926, October 1978.

[8] Christopher M. Brown, Inherent bias and noise in the Hough transform, IEEE Trans. Pattern Anal. Machine Intell., Vol. PAMI-5, No. 5, September 1983.

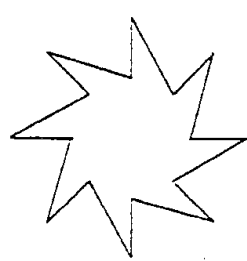

(a)

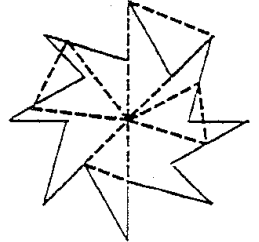

(b)
Figure 1 (a) An 8-fold rotationatly symmetric shape. (b) All the dashed triangles are similar to one another. 

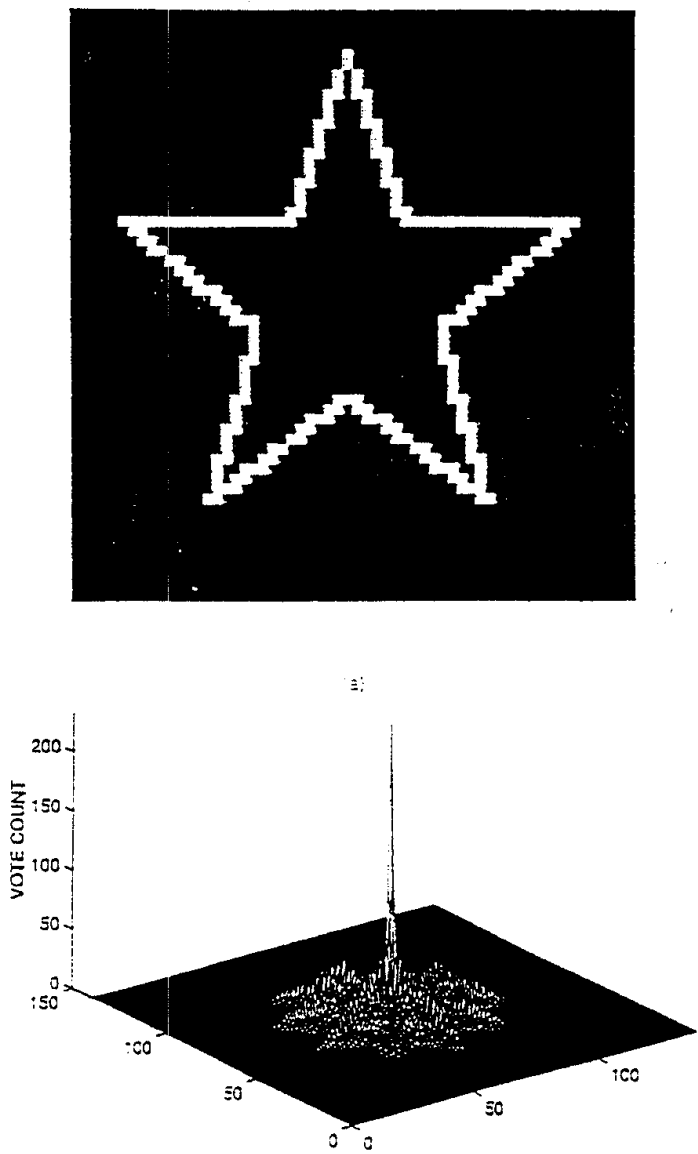

(c)

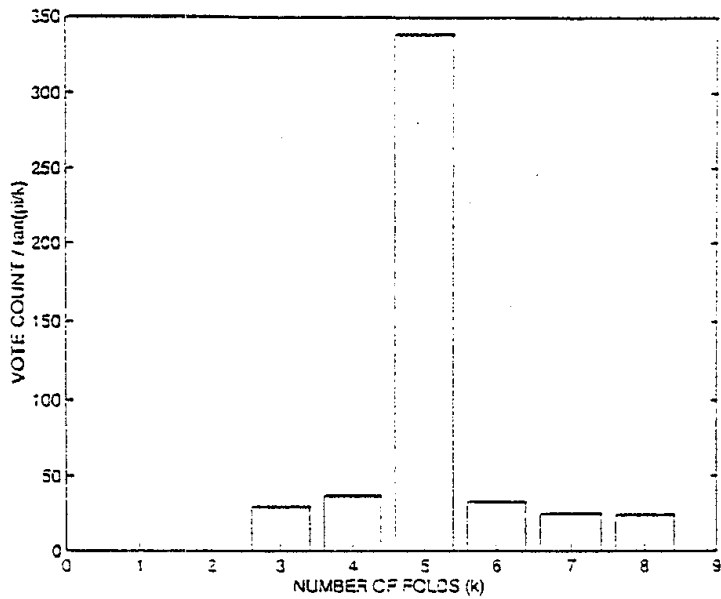

Figure 2(a) The toxso digital imace of a s-fict rotationally symmatre

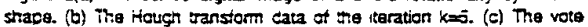
counts of ditierent thratons at the coak pesition of (t).
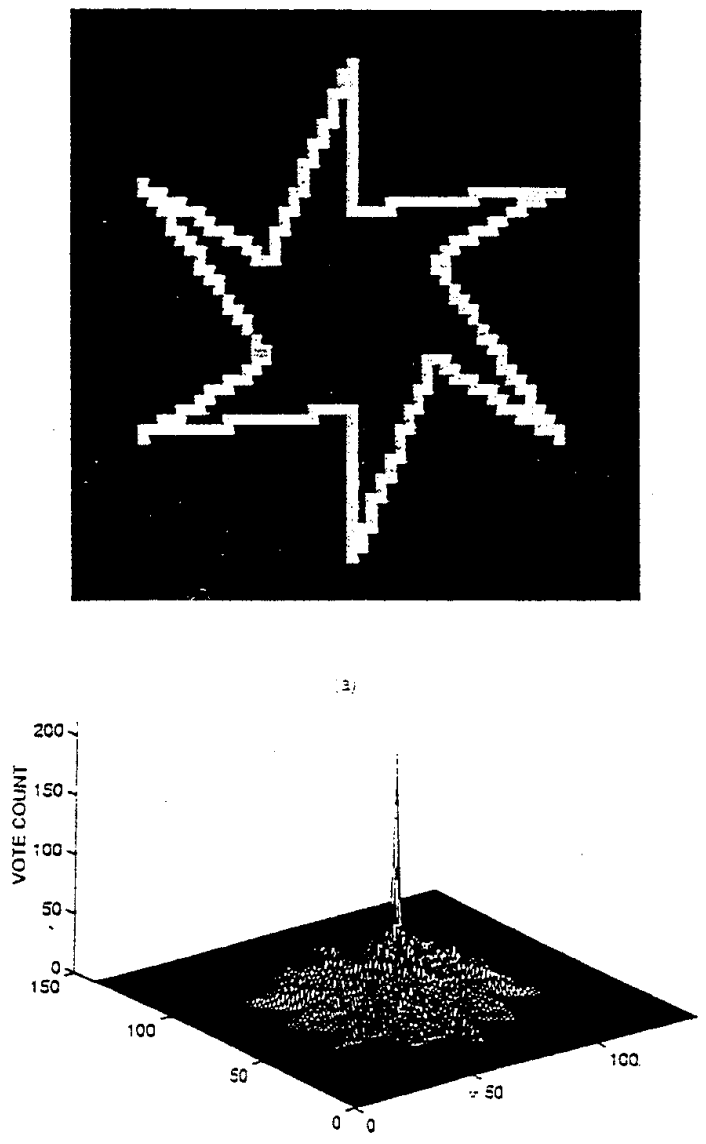

(e)

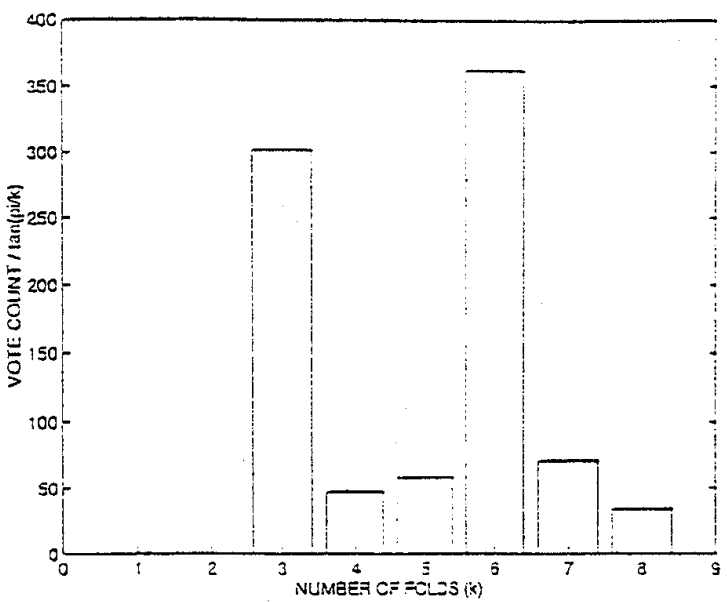

Figure 3(a) the 60Xe0 cigital image st a s-feld retatenally symmatric snape. (b) The Hough iranstom cata $f$ t to teration $k=0$. (c) The veta counts of ditferent therations at to ceax pestien of (o). 


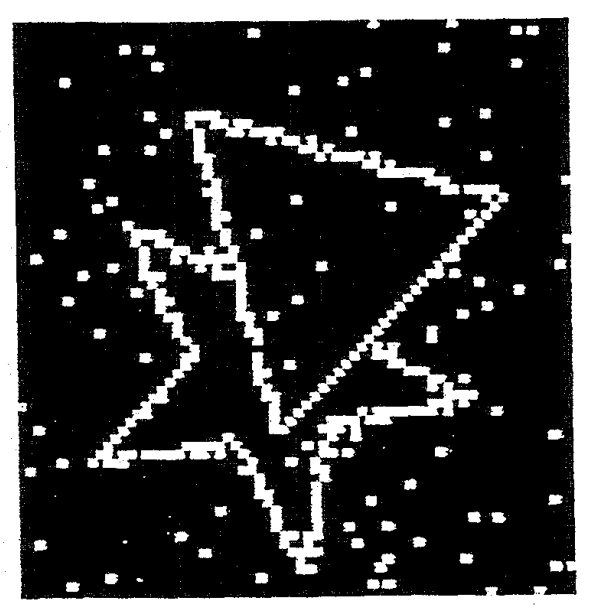

$\vdots$

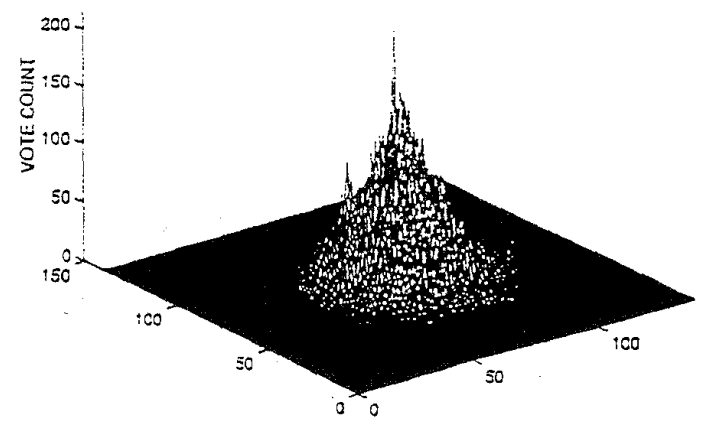

(b)

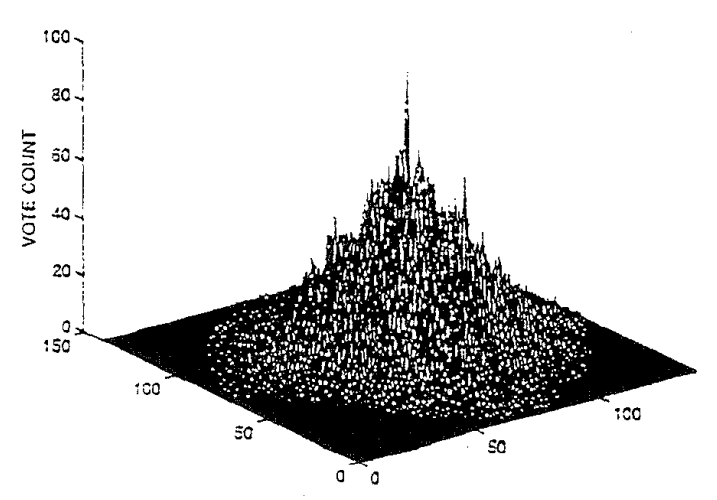

(c)

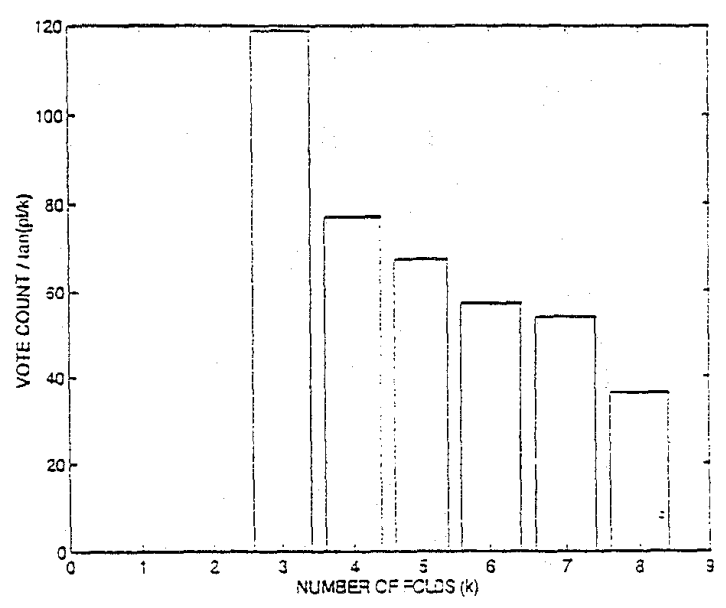

(c)

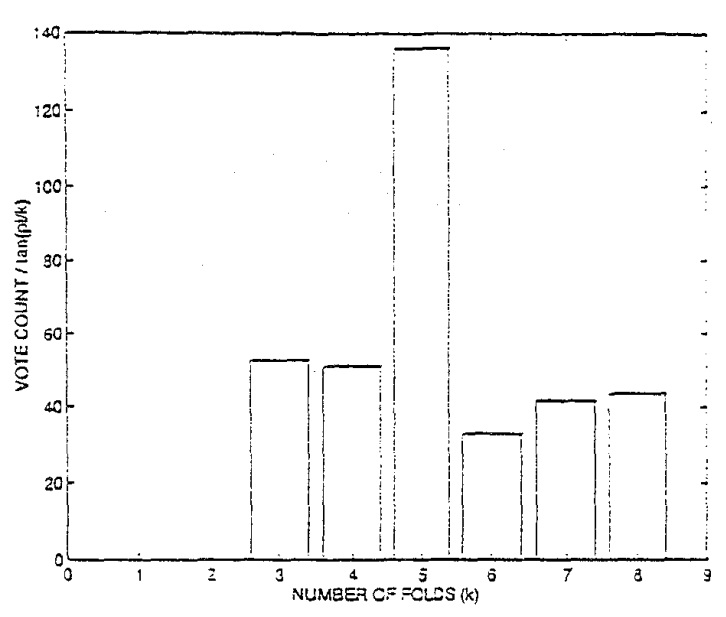

(e)

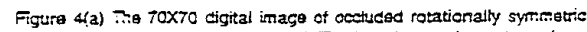
shapes uneer noisy environment. (5) The Hough transtem cats of $5: 0$ iteration $k=5$, (G) The Hough transtom tata of the iteration $k=5$. (C) The counts of everent iterations at the geak cossticn of (c). 